\title{
A Practical New 21 st Century Learning Theory for Significantly Improving STEM Learning Outcomes at all Educational Levels
}

\author{
Stanislaw Paul Maj ${ }^{1,2^{*}}$ (D) \\ 1 SPM Consulting, AUSTRALIA \\ ${ }^{2}$ Assumption University, THAILAND
}

Received 29 August 2021 - Accepted 16 December 2021

\begin{abstract}
Current educational theories, such as Constructivism, are based on subjective principles which are open to interpretation and may result in wide variations in the quality of learning outcomes. Cognitive Load Optimization is a new quantitative, Science of Learning theory. Using this method, it is possible to define schemas (mental patterns of knowledge) with the quantitatively optimized minimum cognitive load which are the basis of instructional material development. Such materials represent the easiest possible learning paths. In one proof of concept experiment this new method was used to teach first year undergraduate mathematics to mature students in online mode. The pass rate and retention rates were both $100 \%$ and all learning objectives were met. Extensive evaluations strongly suggest that Cognitive Load Optimization can significantly improve teaching and learning outcomes in all STEM disciplines at all educational levels - primary and secondary school, college and university.
\end{abstract}

Keywords: cognitive load optimization, cognitive science, learning theories, science of learning

\section{INTRODUCTION}

Teaching practices are based on learning theories that inform educators how students learn. There are different learning theories in use today such as Constructivism, Behaviorism and Cognitivism. The basis of constructivism is that each student constructs their own knowledge (Piaget, 1969). The Behaviorist approach is based on operant conditioning in which positive responses that are reinforced are strengthened and are likely to occur again (Skinner, 1950). Cognitivism is concerned with how information is received, organized, and stored (Happs, 1985). In addition to these theories of learning there exist a wide range of methods and approaches designed to facilitate the educational process such as variation theory (Cheng, 2016), cognitive maps (Diekhoff, 1983), semantic proximities (Nagy, 1984), closeness (Keppens, 2008), attributes (Klausmeirer, 1992), coordinate concepts (Merrill, 1977; Meyer, 1985), structured relationships (Meyer, 1985), elaboration theory (Reigeluth, 1979), etc. However, all of these methods and techniques were developed in the 20th Century and are based on soft science principles. The use of the term "soft" is not pejorative because this type of science is concerned with human behavior which is complex. A soft science approach is useful for evaluating systems that are difficult to measure and hence is used in disciplines such as sociology, psychology, etc. Soft science is qualitative and based on guidelines that can be subjectively interpreted which may result in wide variations in both learning standards and outcomes. In a ten-year period over thirty units in a wide range of STEM subjects offered by seven nationally accredited institutions (two colleges, five universities, including a five-star teaching university) in two countries (Thailand and Australia) were evaluated. STEM subjects included: electrical principles, cyber security, programming, project management, mathematics, etc. In this study unit evaluation criteria included: pass and attrition rates, pedagogical quality, and value for money. Pedagogical quality was evaluated according to both the number of topics taught and the depth of treatment of each topic based on the Structure of the Observed Learning Outcome (SOLO) taxonomy (Biggs \& Collis, 1982). Excluding pre-structural, this taxonomy consists of four levels with associated verbs as evaluation metrics:

4. Extended abstract: Generalize to a new domain.

(c) 2022 by the authors; licensee Modestum. This article is an open access article distributed under the terms and conditions of the Creative Commons Attribution License (http://creativecommons.org/licenses/by/4.0/). 


\section{Contribution to the literature}

- Current learning theories provide subjective guidelines that are open to interpretation. Students are guided to construct their own relational knowledge which may be incomplete or incorrect.

- Cognitive load optimization (CLO) has a simple, quantitative metric for measuring cognitive load. Hence relational knowledge in the form of a schema can be optimally organized. This schema represents the easiest learning path and is given to the students. It is also the basis of instructional development and teaching.

- CLO can result in significant improvements in learning outcomes in STEM subjects at all educational levels and represents a unique, alternative learning theory.

3. Relational: Many interdependent elements i.e., explain, calculate, etc.

2. Multi-structural: Several relevant elements i.e., list, define.

1. Uni-structural: One relevant element.

The goal of learning is to achieve level 3 (and above) which can be assessed by evaluation metrics such as explain, calculate etc. The final exam and unit content were evaluated according to the SOLO levels. For the analyzed units there were considerable variations in both pedagogical quality and pass rates. The pass rates of some units were consistently circa $100 \%$ but at the expense of academic quality, i.e., more complex topics were not taught and also assessments were based on SOLO level 2 metrics such as list, identify etc. In effect high pass rates are not necessarily an assurance of quality learning outcomes as they can be achieved by teaching and/or assessing at a lower SOLO level. Others units had a higher pedagogical standard but in one case pass rates were less than $30 \%$ (Maj, 2021a). The acceptance of low pass rates arguably places learning responsibility on students rather than how the teacher can facilitate better results.

Educational research is important because of the decline in the uptake of STEM disciplines due in part to the perceived difficulty of these disciplines (IET, 2008). Attempts, such as fun activities and experimentally based teaching, to change this perception are reported to have been unsuccessful (Lyons, 2004). Within engineering education, five research areas have been identified including 'Engineering Learning Mechanisms' which are concerned with how to develop knowledge (Education, 2006). In America, the 2012 President's Council of Advisors on Science and Technology reported the need to adopt more empirically validated teaching practices for STEM disciplines (PCAST, 2012).

Educational research faces complexities not found in the physical sciences (Berliner, 2002). A detailed analysis of six exemplar schools concluded (Holkner, 2008):

"It is suggested that there is also a need for more 'scientific' evidence of 'what works in classrooms rather than more qualitative studies'".

\section{Furthermore,}

\begin{abstract}
"As a consequence, there is much criticism of educational research including that it lacks rigor, fails to produce cumulative findings, is theoretically incoherent, ideologically biased, irrelevant to schools, lacks the involvement of teachers, and is poorly communicated and expensive."
\end{abstract}

In order to address these problems global research programs in the science of learning were established.

\section{SCIENCE OF LEARNING}

The American National Science Foundation (NSF), Division of Behavioral and Cognitive Sciences established the Science of Learning (SoL) research agenda with the goals of transformative basic research to advance the SoL with the goal of optimized learning for all (NSF, 2017). A number of research questions were identified such as: "How does the structure of the learning environment impact rate and efficacy of learning? For example, how do timing, content, learning context, development time point, and type of engagement (e.g., active learning, group learning) impact learning processes and outcomes?" In order to translate SoL research into practical implementations the Deans for Impact defined six key questions with the associated cognitive principles and practical implications for the classroom (Impact, 2015). For example, research question \#1, “How do students understand new ideas?" This is based on cognitive principles such as: "students learn new ideas by reference to ideas they already know." Practical implications for the classroom include: "A well sequenced curriculum is important to ensure that students have the prior knowledge they need to master new ideas." Within Australia the Science of Learning Research Centre (SLRC, n. d.) was established and developed twelve PEN principles i.e., Psychology, Education, Neuroscience. For example, PEN principle \#1: "Written text and spoken text don't mix."

Knowledge of how the brain works has been used to identify six learning strategies (spaced practice, interleaving, retrieval practice, elaboration, concrete examples, and dual coding) that can potentially optimize knowledge construction in the learning process (Weinstein, 2018). For example, learning strategy \#2, 
Interleaving (switching between topics while studying) has the application example of "After studying the peripheral nervous system for a few minutes, students can switch to the sympathetic nervous system and then to the parasympathetic system; next time, students can study the three in a different order, noting what new connections they can make between them."

These approaches to improving learning outcomes, based on psychology and neuroscience, whilst of value, are all qualitative. The NSF goal of SoL is optimized learning for all. Optimization can only be achieved if learning theory is quantitatively defined. A quantitative method is important because,

"I often say that when you can measure what you are speaking about, and express it in numbers, you know something about it; but when you cannot measure it, when you cannot express it in numbers, your knowledge is of a meagre and unsatisfactory kind; it may be the beginning of knowledge, but you have scarcely, in your thoughts, advanced to the stage of science, whatever the matter may be." (Thomson, 1889).

\section{COGNITIVE LOAD THEORY}

Cognitive Load Theory (CLT) is based on cognitive science principles that include: schema, Short-Term Memory (STM), Long-Term Memory (LTM), and Intrinsic Cognitive Load (ICL) (Bannert, 2002; Valcke, 2002). Intrinsic Cognitive Load (ICL) is a measure of the complexity of the knowledge to be taught. A schema is a pattern of relationships between elements that confers understanding and is stored in LTM. The process of learning is the construction of schemas in LTM mediated by STM. However, STM (working memory) has only limited capacity and retention time and hence can easily be overloaded by material with a high ICL.

By contrast LTM does not have these handicaps. ICL is a function of the number of elements and their relationships. In this context a useful learning taxonomy is the SOLO taxonomy because it can be used to rank learning outcomes according their relative ICL. Instructional material that is uni-structural (identify) and multi-structural (list) levels have low ICLs because they consist of few elements and interdependencies. However, these represent low-level learning i.e., memory little understanding. By definition relational knowledge consists of many interdependent elements that cannot be understood in isolation. Understanding an element depends upon understanding the context of pre-requisite elements (i.e., relationships). If instructional materials have missing elements, missing relationships and elements not in the correct sequence there are cognitive gaps that contribute to the ICL. The author submits that teaching based on such materials, with cognitive gaps and a high ICL, may force students to come to their own conclusions. The resulting student schema could therefore be incomplete, inconsistent and incorrect resulting in low pass rates - that is why students fail. In essence:

Relational knowledge schema:

- Many interdependent elements that cannot be understood in isolation i.e., high ICL

- Potential cognitive gaps (missing elements and relationships, incorrect sequence of elements) exacerbate ICL

- Overloads STM

- Harder to teach and learn

- Potentially lower pass rates

By contrast, multi-structural knowledge schema:

- Few elements and dependencies i.e., low ICL

- Does not overload STM

- Easier to teach and learn

- Potentially higher pass rates

The goal of learning is relational knowledge i.e., SOLO level 3, which means the student understands how something works hence can explain cause and effect based on the interdependent elements However, measuring ICL in CLT is problematic (de Jong, 2010). Organizing many interdependent elements, in a sequence from the simplest to the most complex and ensuring all elements and their relationships have been defined, is a quantitative combinatorial problem. To address this problem various Neural net models have been used to map relational knowledge. The five-layer semantic cognition model interconnects item, representation, relation, hidden and attribute (Thomas, 2008). The Structured Tensor Analogical Reasoning (STAR) model employs vector-based arguments (Halford, 1998). However, not only are these complex methods that would be challenging to implement but, more importantly they do not solve the problem of combinatorial complexity. The goal of SoL is optimized learning for all. In order to meet this goal what is needed is a simple to use quantitative method for measuring ICL allowing the use of optimization methods that also solves the problem of combinatorial complexity.

\section{COGNITIVE LOAD OPTIMIZATION}

Cognitive Load Optimization (CLO) offers a simple, reliable metric for ICL that also solves the problem of combinatorial complexity. Hence it is possible to convert relational knowledge with a high ICL that overloads STM to knowledge with the lowest possible ICL with no cognitive gaps that does not overload STM. Finally, and importantly, it is a practical and easy to use method. There are three steps to the CLO method.

Step 1: Construct the concept-attribute matrix and optimize for the minimum ICL: Identity and order all concepts, based on attributes, from simple to complex. 
Construct a concept-attribute matrix and optimize (manually or algorithmically) to obtain the minimum ICL. ICL is number of attribute changes divided by the number of concepts the number of concepts.

Step 2: Convert the concept-attribute matrix to a diagram, i.e., simplest possible schema which is given to students: From the concept-matrix a concept-attribute diagram is constructed which is in effect the simplest possible schema. This schema is the easiest learning path and is used as the basis of designing instructional materials, eLearning tools and teaching. The CLO schema is a diagram and hence allows students to concurrently observe concepts and their relationships. A key aspect of CLO is that the optimized schema is given to the students. By contrast in Constructivism students construct their own schema with the potential problems discussed above.

Step 3: Schema is basis designing instructional materials, eLearning tools and teaching: Instructional materials and teaching based on this schema does not overload STM because each concept is presented sequentially to STM from the simplest to the most advanced in a logical, incremental sequence. Because of inheritance each attribute need only be taught once, but is reinforced by the subsequent concept thereby reinforcing existing knowledge. Learning new knowledge is therefore automatically contextualized. In effect CLO converts a complex relational knowledge schema with a high ICL to a relational knowledge schema with the lowest possible IC hence meeting the SoL goal of optimized learning for all.

\section{Implementation Example \#1 - Mathematics}

Consider the sinusoidal form $A \sin (\omega t \pm \alpha)$. This equation has multiple interdependent attributes i.e., elements and hence a high ICL.

\section{Step 1: Construct the concept-attribute matrix and optimize for the minimum ICL}

For this equation identify all attributes i.e., degrees, radians, Amplitude (A), Lag/Lead (L), cycle period $(\rho \theta)$ and cycle time $(\omega t)$. Attributes may have different characteristics - quantitative or qualitative (discrete or continuous), binary, multistate etc. In this example the attributes are only binary. Attributes define concepts. The objective is to order concepts, based on their attributes, into the simplest arrangement with the lowest ICL. With relatively small data sets, such as this one, optimization can be performed manually. For larger data sets optimization algorithms can be used. Illustratively, this example was optimized algorithmically resulting in possible arrangements from the highest ICL to the optimally minimum ICL. In this example the minimum ICL was found to be number of attribute changes (8) divided by the number of concepts the number of concepts (5) i.e., 1.6 (Table 1).
Table 1. Concept attribute matrix-1

\begin{tabular}{|c|c|c|c|c|c|c|}
\hline Attribute & Deg & Rad & $\mathrm{A}$ & $\mathrm{L}$ & $p \theta$ & $\omega t$ \\
\hline \multicolumn{7}{|l|}{ Concept } \\
\hline 0 . sine wave (prerequisite) & 0 & 0 & 0 & 0 & 0 & 0 \\
\hline 1. $y=\sin \theta$ & 1 & 1 & 0 & 0 & 0 & 0 \\
\hline 2. $y=A \sin \theta$ & 1 & 1 & 1 & 0 & 0 & 0 \\
\hline 3. $y=A \sin (\theta \pm L)$ & 1 & 1 & 1 & 1 & 0 & 0 \\
\hline 4. $y=A \sin (p \theta \pm L)$ & 1 & 1 & 1 & 1 & 1 & 0 \\
\hline 5. $y=A \sin (\omega t \pm L)$ & 0 & 1 & 1 & 1 & 0 & 1 \\
\hline $\mathrm{ICL}=8 / 5=1.6$ & & & & & & \\
\hline
\end{tabular}

These optimally ordered attributes define five equations representing five concepts based on the simple concept of a sine waveform (concept 0). Inheritance controls combinatorial complexity. Degrees and radians are the simplest attributes and would be taught first. Concept 1 has the attributes of degrees and radians. Concept 2 inherits these two attributes but has the additional attribute of Amplitude (A) i.e., a predecessor/successor relationship. Concept 3 has the same three attributes as concept 2 but with the additional attribute of Lag/Lead (L). Concept 3 is therefore defined by four attributes three of which were inherited. Branching occurs when advanced concepts do not have common attributes. The advanced concept 4 inherits four attributes (degrees, radians, $\mathrm{A}$ and $\mathrm{L}$ ), however concept 5 only inherits three attributes (radians, A and L). In effect an attribute reversal for degrees because it is no longer applicable. Also concepts 4 and 5 diverge according to attributes $(\rho \theta)$ and $(\omega t)$ which distinguish between the cycle period in angles $(\mathrm{P})$ or time $(\mathrm{T})$ i.e., $\mathrm{x}$ axis can be either $\theta$ or $t$. This divergence is more evident in the concept-attribute diagram. The ICL of this optimized matrix is 1.6 .

\section{Step 2: Convert the concept-attribute matrix to a diagram i.e., simplest possible schema}

Using the optimized concept-attribute matrix, with the lowest possible ICL, construct the concept attribute diagram i.e., simplest possible schema, which is given to the students (Figure 1).

$$
\begin{array}{ll}
y=A \sin (p \theta \pm L) & y=A \sin (\omega t \\
P=\text { cycle } / p & T=\text { cycle } / \omega
\end{array}
$$

Cycle Period Angle (P)

Cycle Period Time (T)

Figure 1. Optimized schema-1 
Table 2. Concept attribute matrix-2

\begin{tabular}{lcccccccc}
\hline Attribute & $\begin{array}{c}\text { Shared } \\
\text { medium }\end{array}$ & Trans & Rec & B/cast & $\begin{array}{c}\text { CSMA/ } \\
\text { CD }\end{array}$ & $\begin{array}{c}\text { Half } \\
\text { duplex }\end{array}$ & $\begin{array}{c}\text { MAC } \\
\text { address table }\end{array}$ & Unicast \\
Concept & 1 & 1 & 1 & 1 & 1 & 1 & 0 & 0 \\
duplex
\end{tabular}

\section{Step 3: Schema is basis designing instructional materials, eLearning tools and teaching}

The schema (Figure 1) represents the easiest learning path and is used for the development of instructional materials and teaching.

\section{Implementation Example \#2 - Network Technology}

Hubs and switches are network technology devices. They have attributes that define their operation such as half-duplex, full-duplex, collision prevention mechanisms, etc.

\section{Step 1: Construct the concept-attribute matrix and optimize for the minimum ICL}

The ICL for the optimized matrix is attribute changes (5) divided by number of concepts (2) i.e., 2.5 (Table 2).

\section{Step 2: Convert the concept-attribute matrix to a diagram i.e., simplest possible schema}

A schema, based on the optimized matrix can be constructed and represents a logically structured teaching sequence from the simplest to the most complex concept, i.e., easiest learning sequence (Figure 2).

\section{Step 3: Schema is basis designing instructional materials, eLearning tools and teaching}

Both devices have common attributes: shared medium, transmit, receive, and broadcast. A hub has two unique attributes: collision prevention with the CSMA/CD protocol and half duplex. A switch has three unique attributes: collision prevention based on a MAC address table, unicast, and full duplex. Based on this

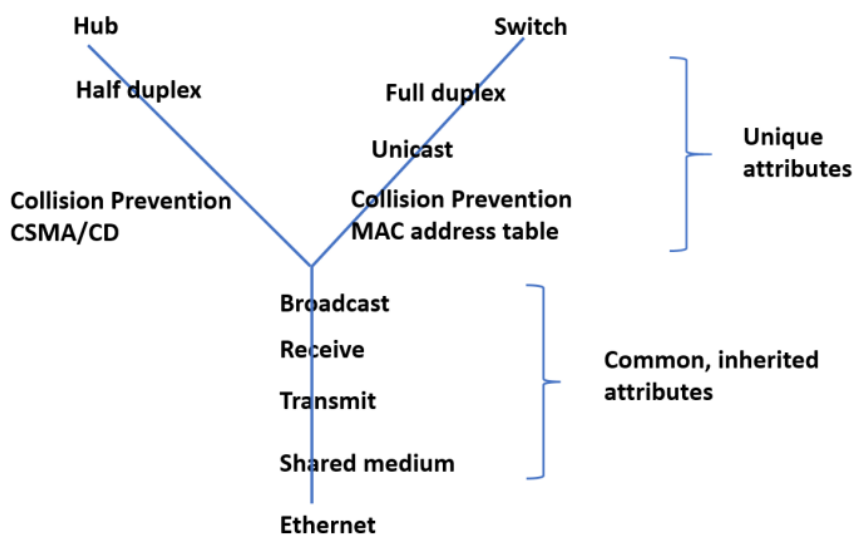

Figure 2. Optimized schema-2 optimized CLO schema eLearning tools (called State Model Diagrams - SMDs) can be developed for a hub and a switch. Illustratively, the single eLearning tool for a switch can be used to teach not only all common attributes (transmit, receive and broadcast) but also all unique attributes of a switch (Figure 3). The MAC address table establishes a direct connection between the transmitting PC to the receiving PC allowing unicast to occur. The diagram can also be used to explain full duplex.

Importantly more advanced switch technologies, such as Virtual LAN (VLAN), EtherChannel, Spanning Tree Protocol (STP), etc., may be added to the conceptattribute matrix. In effect the optimized schema may be used for teaching at secondary school level, but extended to teaching at higher levels such as college and undergraduate levels.

\section{CLO EVALUATIONS}

Published work has demonstrated that CLO is applicable to STEM disciplines in all educational sectors (school, college and university) and can result in significant improvements in learning outcomes. CLO has been successfully used in a wide range of STEM disciplines that include: engineering drawing, cybersecurity, project management, electrical principles, industrial applications of IT, network engineering, computer systems engineering, science, biomedical engineering, object-oriented programming, etc. and when fully implemented both the pass rates and retention rates were high (asymptotic to 100\%) along with very high student satisfaction feedback (Maj, 2020).

\section{University Sector - Undergraduate}

\section{Mathematics - statistical proof of concept analysis}

In a small, proof-of-concept experiment two topics sinusoidal form $A \sin (\omega t \pm a)$ and simple navigation were taught based on two different learning methods standard method and CLO - and the results evaluated. For both experiments data was statistically validated using the Analysis of Variance (ANOVA) model. Given the small sample sizes the Repeated Measures Mixed Models methods was also used. For the mathematics experiment learning was evaluated, without student access to study materials, by randomized questions at three ICL levels (1 low ICL, 2 medium ICL, 3 high ICL) representative of increasing levels of complexity. For 


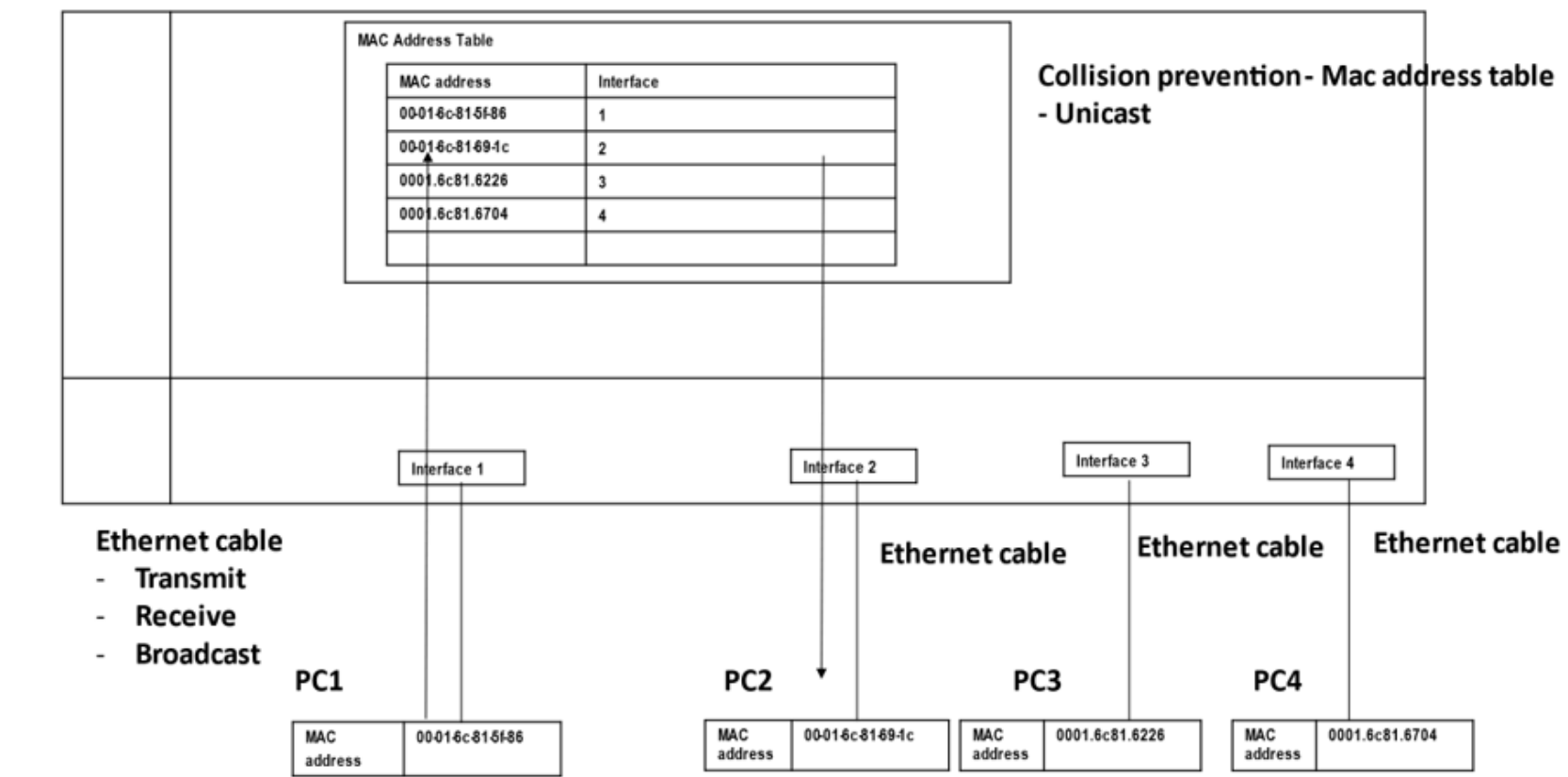

Figure 3. eLearning tool for switch

CLO based teaching there was a statistically significant improvement when comparing the question type 3 to 2 and 3 to 1 . In the navigation experiment learning was evaluated, without access to study materials, by randomized questions, at four ICL levels (1 low ICL, 2 medium ICL, 3a high ICL and $3 b$ higher level ICL) representative of increasing levels of complexity. For CLO based teaching there was a statistically significant improvement at level $3 \mathrm{a}$ with the level of significance increasing from $3 a$ to $3 b$ (Maj, 2020). In conclusion, the use of CLO improves learning outcomes at SOLO level 3 i.e., relational knowledge.

\section{Mathematics unit - remote online learning}

A first-year engineering mathematics unit based on CLO was used to teach remote, online students. All the students were employed in professional roles hence working full time, studying part time (time poor) and mainly resident in developing countries with poor and sometimes unreliable internet connectivity. During the lectures students were restricted to using a simple textonly chat line that could not be used to enter mathematical symbols. Commensurate with a first-year undergraduate level, all topics were taught to the standard required. Significantly there was zero attrition and all students passed. Feedback was sought from all participants by means of simple Likert based questions (scale 1 to 5 ) with the opportunity to provide comments. Every participant recorded a score of 5 (highest) for every question. Comments such as the following were received:
"I found our Monday tutorial sessions to be the perfect blend of instructional teaching, challenging questions and most importantly thorough explanation. So far this math unit is the best unit I have ever been in ever, I thoroughly enjoy our teaching and methods with interaction to make sure we are all learning. I also really enjoy how you can keep students active in the topic to help learning, and not like many other teachers, including one I had to drop out recently, that just read the content and answer questions quite vaguely. I would personally love if you could teach more units or if XXX could possibly get other teachers to adopt your teaching curriculum method." (Maj, 2018).

\section{Programming unit}

The CLO method has been evaluated as the basis of teaching introductory Object-Oriented Programming (OOP) to undergraduates. In this experiment $50 \%$ of the instructional material was rewritten based on CLO and taught on one campus and the standard material, based on Greenfoot, was used on two other campuses concurrently. Greenfoot is an integrated development environment with extensive educational resources that includes a prescribed textbook designed for teaching school children. Greenfoot has two high-level design goals - make programming engaging, creative and satisfying; help in teaching important universal programming concepts. The second high-level objective is concerned with supporting teaching with specific requirements that include avoiding cognitive overload 
(Kolling, 2010, 2016). However, a CLO analysis found the material to have a very high ICL. At the two campuses based $100 \%$ on Greenfoot, with cohort sizes of 50 and 56 , the pass rates were $28 \%$ and $27 \%$ respectively. At the third campus, with $50 \%$ of the unit material based on the CLO method, with a cohort size of 20 the pass rate was $45 \%$ representing a $60 \%$ improvement. It should be noted this was a double-blind experiment in which the CLO instructor did not know the final exam questions and did not mark the final exam papers. It is submitted that if all of the material was based on the CLO method the pass rate would have been asymptotic to $100 \%$ (Maj, 2020).

\section{Network technology unit}

CLO was used as the basis of teaching graduate, overseas, onshore students enrolled on an MSc IT conversion course. The students were graduates in a range of non-IT disciplines such as Biology, Mechanical engineering etc. They were taught all the material from the Cisco Certified Network Associate (CCNA) award that was rewritten based on CLO and given 24 hours of face-to-face instruction including laboratory time. Leaning outcomes were evaluated against Cisco CCNA students who received over 100 hours of instruction. The learning outcomes of the postgraduate student cohort were significantly better than the Cisco based students and comparable to a qualified and experienced expert. Student learning of the CLO based cohort was evaluated six weeks after completion of their final exam. It was found that all students were able to correctly answer complex questions and answered them using the diagrammatic SMD method. It was noted that in most cases the diagrams were not identical to those taught. This is indicative that the students developed their own schema resident in long term memory (Maj et al., 2005).

\section{STEM for business students - remote online learning}

A degree in Business IT or IT management requires students to study units in STEM technical subjects such as computer and network technology, IT infrastructure, cybersecurity etc. Business students are unlikely to have an appropriate technical background. The challenge is not to make business students technical experts - this is neither desirable or possible. Rather the objective is to provide business students with sufficient technical knowledge appropriate to administrative/management roles in the workplace and the confidence to interact with discipline experts. Four STEM based business units (cybersecurity, cybersecurity management, information systems management, and management information systems) at two different universities in two different countries were analyzed and found the material was almost exclusively taught as technical lists with little or no explanations. Using CLO theory all four STEM based business units were analyzed and optimized as a sequence of three lectures (computer technology, network technology and security) that were remotely given to a cohort of 33 business students whose first language was not English. Verbatim comments regarding their previous experience learning STEM based subjects were all negative such as: "It doesn't have explaining in each, how it works." In response to the question would you remember what you were taught in three months' time all comments were negative such as, "Not remember at all." In conclusion $45 \%$ of students positively rated this method. By contrast $100 \%$ of the students rated the CLO method of teaching either very good or excellent. Furthermore, all verbatim comments were positive such as: "Able to understand the concept and how does it work. In this way you will be able to identify the issues when it happens because you are understand how does it work". In response to the question, would you remember what you have been taught in three months' time all verbatim comments were positive such as: "Sure, I prefer this method because I can have a full understanding. I like the way I can think of something not remember it". This student comment is highly significant because it strongly suggests complex relational knowledge is likely to be resident in LTM - the goal of learning (Maj \& Nuangjamnong, 2020).

Perceptions of the use of CLO to teach STEM based subjects to business students was evaluated using the Technology Acceptance Model (TAM) and the Unified Theory of Acceptance and Use of Technology (UTAUT) frameworks. The results from a cohort of 210 participants found that CLO would substantially improve their learning performance. (Nuangjamnong \& Maj, 2022).

\section{College Sector}

The Cisco Network Academy is a global IT and cybersecurity education program with 9,000 Cisco accredited academies, 20,000 Cisco qualified instructors in 170 countries (Academy). Within the Australian college sector, the professional Cisco Certified Network Professional (CCNP) is often the basis of their Diploma award. The pre-requisite to the CCNP is the Cisco Certified Network Associate (CCNA) award. A cohort of students who were in the process of completing their CCNP award were given a one-hour tutorial on a single topic (Spanning Tree Protocol) using only the SMD eLearning tool. Feedback from these students was as follows:

Student 1: Yes, I have learnt more in this (one hour) period than the whole of the semester.

\section{Student 2: Excellent, far clearer than any Cisco material.}

Student 3: Yes! the diagrams illustrate the process in a very easy to understand format to allow the subject to be learnt. 
Student 4: Nice to have a conceptual model to aid understanding.

\section{Secondary School Sector}

\section{CLO-based eLearning tools}

The efficacy of eLearning tools and technologies has been questioned. An analysis of the use of innovative learning technologies in exemplar schools found,

"We know that after nearly five decades of computers in education there is still confusion about the use of technology in classrooms and widespread reluctance to move beyond tokenistic use. There is not a universal, shared vision regarding the use of technology in the classroom and teachers are confronted with an eclectic array of theories and instructional designs and bombarded with confusing, even romantic views of what the technology is capable of delivering. We also know that is it not possible to definitely establish a direct link between learning with technology and improved outcomes." (Holkner et al., 2008).

Based on the optimized schema, eLearning tools can be developed. The CLO derived eLearning tools for IT, called State Model Diagrams (SMDs), captures all the key learning concepts and their relationships diagrammatically and provides,

"An overview of the entire network or increasing levels of detail may be obtained while maintaining links and interfaces between the different levels. Furthermore, SMDs allow technical detail to be introduced in an integrated and controlled manner, thereby supporting student learning at both introductory and advanced levels. In effect, as student's progress they do not have to learn a new conceptual model; rather they can build upon and extend their existing knowledge." (Maj \& Veal, 2007).

Hence, CLO derived eLearning SMDs support student learning at all educational levels.

\section{IT - proof of concept}

Within Australia a recommended textbook for teaching IT to 12-15-year-old students was analyzed for the topics of hub and switch (Grover \& Winton, 2017). The material was found to have a high ICL. For example, according to the textbook,

"Switches work by keeping a table of addresses for each connected device. Each device has its own network interface card (NIC) that is numbered with a media access control address, known as a
MAC address. MAC addresses are also known as Ethernet addresses."

Cognitive gaps: How does a switch identify which device is on which interface? What is Ethernet? Ethernet not explained until later in the book. What devices use CSMA/CD? Both hub and switch?

Teaching materials based on CLO eLearning State Model Diagram tools such as Figure 3 were used as the basis of teaching hub and switch technology to a cohort of 21 students that attended a one-day workshop. In this proof-of-concept experiment was independently monitored who concluded: Throughout the exercise students were fully engaged; learning occurred and did not exceed their abilities; all learning objectives were met (Maj, 2021b).

\section{IT - extended study}

CLO was used to develop instructional materials and evaluated by teaching a cohort of twenty 14-15-year-old students who attended nine one-day session on a university campus using a dedicated network teaching laboratory. The objective was to teach PC to PC connectivity on different networks via different LAN technologies (hub, switch, wireless). In this experiment students had to learn not only many different concepts and associated technologies but also how to connect, configure, test and fault find connectivity using PCs, hubs, switches, wireless access points and routers. This includes using the complex Command Line used for switches, wireless access points and routers. Instruction, practical exercises and learning evaluations were based on CLO schemas and associated CLO derived SMD eLearning tools which represent all attributes and concepts. They allowed knowledge to be represented with increasing or decreasing levels of detail whilst maintaining relational links. At the conclusion of the nine-week study, student learning as evaluated as follows:

1. After configuring two PCs on the same network using a switch and wireless access point students were asked to complete blank SMDs for a switch and wireless access point.

2. After configuring two PCs on the same network connected by either a hub, switch or wireless access point, students were asked to complete a blank SMD template.

3. After configuring two PCs on different networks by a router using either hubs, switches or wireless students were asked to complete a blank SMD template.

4. Provided with information from frame capture, students were asked to complete the associated SMD template. This was done for two PCs connected together on the same network and also 
two PCs on different networks connected by a router.

5. Provided with a partially competed SMD template, students were required to complete the diagram.

6. Students were given questions such as, what does ARP stand for, why is it needed and how does it work?

These questions were designed to evaluate relational knowledge, i.e., the ability to analyze and explain which is the objective of learning. For evaluations $1,2,3,5$, and 6 all students scored $100 \%$. However only two students successfully completed evaluation 4 . Students found it difficult to both use frame capture and interpret the results. No evaluation was conducted to determine if knowledge was stored in LTM (Maj, 2021c).

\section{Primary School Sector}

\section{Proof of concept - science education}

Australian textbooks for 12-to14 year old students were analyzed for the topic of energy (Thickett, 2012; Williamson, 2011, 2020). Using CLO it was found that this material has a high ICL and cognitive gaps. Based on the CLO method, a single optimized, extended abstract schema was created for the science of energy. Extended abstract is the highest SOLO level (i.e., 4) and represents learning that goes beyond the immediate concept making links to other concepts. The evaluation metrics are generalizing, predicting etc. According to Hattie (2004),

\section{"From multi-structural to relational. This involves more that 'getting to know more about a topic or being adept at following through a sequence of procedures; it includes understanding or integrating what is known into a coherent system where the parts are inter-related. This inter- relationship comes about as a result of an ability to form an over viewing principle which can be derived from the information given.}

From relational to extended abstract. This process requires dedicated hard work to master abstract concepts and relationships which allows the student to derive more generalized principles and transfer understanding to new tasks and situations."

To reiterate, current learning theories, such as Constructivism, provide materials and instruction in order to guide students to acquire, for example, relational knowledge i.e., an understanding based on a coherent system of inter-related parts. This may not only be inefficient but also potentially error prone i.e., trial and error learning. The resulting student schema's may be incomplete and/or incorrect. In the case of acquiring extended abstract knowledge the problem is potentially compounded as this requires 'dedicated hard work' by students. By contrast, in the CLO method, instructional materials and teaching are based on optimized relational knowledge schema's which represent the easiest learning paths. The optimized schema's are given to students. In the CLO method learning then consists of internalizing the schema's. Likewise, CLO defines optimized extended abstract schema's (SOLO level 4) which are given to students. Hence this arguably does not require dedicated hard work by students. The CLO level 4 (extended abstract) schema:

- Represents the easiest possible learning path,

- Is applicable to all forms of energy i.e., transfer of understanding,

- Learnt once but applied to all types of energy,

- Contextualizes new concepts, and

- Reinforces previously acquired knowledge.

Using this schema and associated teaching materials, science concepts normally taught to 12-14-year-old students were successfully taught to an 8-year-old student. The schema was used to teach one type of Potential energy source (food, Joules) and the associated Kinetic energy conversion (motion, Joules per second). The same schema was used to include different types of potential energy (food, fuel, batteries etc.) and kinetic energy (motion, light etc.) and their relationship. The student had pre-requisite knowledge of concepts such as weight ( $g$ and $\mathrm{kg}$ ), volume (l) etc., and the mathematical operations of division and multiplication. Further work is needed. Significantly this extended abstract schema can also be used to include and therefore contextualize other, more advanced, aspects of energy. For example, battery kinetic energy (Joules per second) can also be represented in terms of voltage and current $(\mathrm{P}=\mathrm{IV})$. A prerequisite would of course be to teach Ohm's Law.

\section{CONCLUSIONS}

In cognitive science, knowledge is represented by a schema resident in Long Term Memory. There are different categories of knowledge with relational knowledge conferring the ability to explain causes. In the learning theories in use today, the role of the teacher is to facilitate the acquisition of relational knowledge schemas. However relational knowledge is a complex chain of inter-related elements the complexity of which is called the Intrinsic Cognitive Load. During the learning process, missing elements or relationships may result in relational knowledge with a high cognitive load i.e., the schema is incomplete, inconsistent or incorrect. That is why students fail. The goal of the Science of Leaning is optimized learning for all, which can only be achieved using quantitative methods. In cognitive science, measuring cognitive load has proved problematic. Cognitive Load Optimization (CLO) 
provides a simple quantitative metric for cognitive load and hence it is possible to define relational knowledge schemas that have the optimized minimum intrinsic cognitive load. This optimized schema represents the easiest learning path. Importantly this optimized schema is not only the basis of instructional development, teaching and the development of eLearning tools but it is given to students. A proof-of-concept experiment suggest that CLO can also model extended abstract knowledge - the highest SOLO level of knowledge. There is a body of published results indicative that CLO is applicable to STEM disciplines at all educational levels and when applied can result in significant improvements in learning outcomes. However, further work is needed. CLO offers an alternative approach to STEM educators.

Author contributions: All authors have sufficiently contributed to the study, and agreed with the results and conclusions.

Funding: No funding source is reported for this study.

Declaration of interest: No conflict of interest is declared by authors.

\section{REFERENCES}

Bannert, M. (2002). Managing cognitive load - recent trends in cognitive load theory. Learning and Instruction, 12(1), 139-146. https://doi.org/ 10.1016/S0959-4752(01)00021-4

Berliner, D. C. (2002). Educational research: The hardest science of all. Educational Researcher, 31(8), 18-20. https:// doi.org/10.3102/0013189X031008018

Biggs, J. B., \& Collis, K. F. (1982). Evaluating the quality of learning: The SOLO taxonomy (Structure of the observed learning outcome). Academic Press.

Cheng, E. W. L. (2016). Learning through variation theory: A case study. International Journal of Teaching and Learning in Higher Education, 28(2), 283292.

de Jong, T. (2010). Cognitive load theory, educational research, and instructional design: some food for thought. Instructional Science, 38, 105-134. https: / / doi.org/10.1007/s11251-009-9110-0

Diekhoff, G. M. (1983). Relationship judgements in the evaluation of structural understanding. Journal of Educational Psychology, 75, 227-233. https:/ / doi.org/10.1037/0022-0663.75.2.227

Education. (2006). The research agenda for the new discipline of engineering education. Journal of Engineering Education, 95(4), 259-261. https://doi. org/10.1002/j.2168-9830.2006.tb00900.x

Grover, D., \& Winton, S. (2017). Digital technologies for the Australian curriculum. A project-based approach for years 7 and 8. Cengage.

Halford, G. S., Wilson, W. H., \& Phillips, S. (1998). Processing capacity defined by relational complexity: Implications for comparative, developmental, and cognitive psychology. Behavioral and Brain Sciences, 21(6), 803-831. https:/ / doi.org/10.1017/S0140525X98001769

Happs, J. C. (1985). Cognitive learning theory and classroom complexity. Research in Science and Technology Education, 3, 159-174. https://doi.org/ 10.1080/0263514850030109a

Hattie, J. (2004). Cognitive processes in AsTTle: The SOLO taxonomy. Ministry of Education.

Holkner, B., Romeo, G., Henderson, M., Auld, G., Russell, G., Seah, W. T., \& Fernando, A. (2008). Exemplar schools using innovative learning technologies. https://research.monash.edu/en/ publications / exemplar-schools-using-innovativelearning-technologies

IET. (2008). Studying STEM: What are the barriers. https:/ / studylib.net/doc/10501523/studyingstem--what-are-the-barriers \%3F-factfilesI

Impact. (2015). The science of learning. https:/ / deansforimpact.org/resources/thescience-of-learning/

Keppens, J., \& Hay, D. (2008). Concept map assessment for teaching computer science programming. Computer Science Education, 18(1), 31-42. https:/ / doi.org/10.1080/08993400701864880

Klausmeirer, H. J. (1992). Concept learning and concept teaching. Educational Psychologist, 27(3), 267-289. https:/ / doi.org/10.1207/s15326985ep2703_1

Kolling, M. (2010). The greenfoot programming environment. ACM Transactions on Computing Education, 10(4), 1-21. https://doi.org/10.1145/ 1868358.1868361

Kolling, M. (2016). Introduction to programming with greenfoot. Pearson.

Lyons, T. (2004). Choosing physical science courses: The importance of cultural and social capital in enrolment decisions of high achieving students [Paper presentation]. IOSTE X1 Symposium, Lublin, Poland.

Maj, S. P. (2018). Cognitive load optimization - A new, practical, easy-to-use method for enhancing STEM educational outcomes based on the science of learning [Paper presentation]. 2018 IEEE International Conference on Teaching, Assessment, and Learning for Engineering, Wollongong, Australia. https://doi.org/10.1109/TALE.2018.8615324

Maj, S. P. (2020). Cognitive load optimization - A statistical evaluation for three STEM disciplines [Paper presentation]. IEEE International Conference on Teaching, Assessment, and Learning for Engineering, Takamatsu, Japan. https://doi.org/ 10.1109/TALE48869.2020.9368430

Maj, S. P. (2021a). Benchmarking educational quality - An independent analysis and alternative approach [Paper 
presentation]. 38th International Conference on Innovation, Practice \& Research in the use of Educational Technologies in Tertiary Education, On line. https://doi.org/10.14742/ascilite2021. 0144

Maj, S. P. (2021b). A new 21st century quantitative learning theory for improving STEM education in both face to face and online modes [Paper presentation]. STEM 2021, Vancouver, Canada.

Maj, S. P. (2021c). World class STEM - Benchmarking and delivering based on evidence based cognitive science [Paper presentation]. IEEE International Conference on Teaching, Assessment, and Learning for Engineering, Wuhan, China.

Maj, S. P., \& Nuangjamnong, C. (2020). Using cognitive load optimization to teach STEM disciplines to business students [Paper presentation]. IEEE International Conference on Teaching, Assessment, and Learning for Engineering, Takamatsu, Japan. https:/ / doi.org/10.1109/TALE48869.2020.9368351

Maj, S. P., \& Veal, D. (2007). State model diagrams as a pedagogical tool - An international evaluation. IEEE Transactions on Education, 50(3), 204-207. https://doi.org/10.1109/TE.2007.900028

Maj, S. P., Kohli, G., \& Fetherston, T. (2005). A pedagogical evaluation of new state model diagrams for teaching internetwork technologies [Paper presentation]. 28th Australasian Computer Science Conference, Newcastle, Australia.

Merrill, M. D., \& Tennyson, R. D. (1977). Teaching concepts: An instructional design guide. Educational Technology Publications.

Meyer, B. J. F. (1985). Signalling the structure of text. In D. H. Jonassen (Ed.), Technology of text (Vol. 2). Educational Technology Publications.

Nagy, P. (1984). Cognitive structure and the spatial metaphor. In N. P. (Ed.), The representation of cognitive structure. Ontario Institute for Studies in Education.

NSF. (2017). Science of learning. https://www.nsf.gov/ funding/pgm_summ.jsp?pims_id=5567

Nuangjamnong, C., \& Maj, S. P. (2022). Students behaviour intention to adopt cognitive load optimization to teach STEM in graduate studies. Journal of Education Naresan University, 24(3).

PCAST. (2012). Engage to excel: Producing one million additional college graduates with degrees in science, technology, engineering, and mathematics. https:/ / obamawhitehouse.archives.gov/sites/def ault/files/microsites/ostp/pcast-engage-to-excelfinal_2-25-12.pdf

Piaget, J. I. B. (1969). The psychology of the child. Basic Books.

Reigeluth, C. M. (1979). In search of a better way of organising instruction: The elaboration theory. Journal of Instructional Development, 2, 8-15. https:/ / doi.org/10.1007/BF02984374

Skinner, B. F. (1950). Are theories of learning necessary. Psychological Review, 57, 193-216. https://doi.org/ $10.1037 /$ h0054367

SLRC. (n. d.). Science of learning research centre. https://www.slrc.org.au/

Thickett, G., \& Stamell, J. (2012). Science study guide year 8. Get the results you want. Pascal Press.

Thomas, M. S. C., \& McClelland, J. L. (2008). Connectionist models of cognition. In Cambridge handbook of computational psychology (pp. 23-58). Cambridge University Press. https://doi.org/ 10.1017/CBO9780511816772.005

Thomson, W. (1889). Electrical units of measurements. In Popular lectures and addresses (pp. 73-136). Cambridge University Press. https://doi.org/ 10.1017/CBO9780511997242.006

Valcke, M. (2002). Cognitive load: Updating the theory. Learning and Instruction, 12(1), 147-154. https:/ / doi.org/10.1016/S0959-4752(01)00022-6

Weinstein, Y., Madan, C. R., \& Sumeracki, M.A. (2018). Teaching the science of learning. Cognitive Research: Principles and Implications, 3, 2. https://doi.org/ 10.1186/s41235-017-0087-y

Williamson, K., \& Garton, A. (2011). Science essentials 8. MacMillan.

Williamson, K., \& Garton, A. (2020). Science essentials 8 (Australian curriculum ed.). Colin McNeil.

\section{http://www.ejmste.com}

